\title{
Transformación personal y social en el ámbito de la salud a través de la educación: Breve recorrido desde la sabiduría indígena ancestral hasta la tiranía moderna de lo saludable
}

\author{
Personal and Social Transformation in the Health Area through Education: A Brief \\ Journey from the Ancestral Indigenous Wisdom to the Modern Tyranny of Healthiness
}

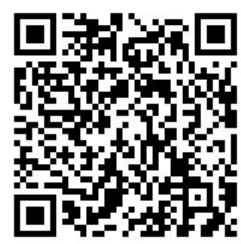

Andrés de Muller ${ }^{1}$

Universidad de la Salle

San José, Costa Rica

ademuller@yahoo.com

Recibido 20 de noviembre de 2014 • Corregido 2 de mayo de 2015 • Aceptado 31 de julio de 2015

Resumen. El artículo busca una reivindicación de la sabiduría ancestral de los pueblos indígenas en el ámbito de la salud, analizando cómo esta ha sido mercantilizada por intereses de grandes compañías (entre las que destacan las relacionadas con la industria farmacéutica) en detrimento de una concepción holística del bienestar a través de la educación. Por otra parte, el concepto de salud como derecho entra en conflicto con su comercialización al mejor postor, incidiendo en la deshumanización de los servicios sanitarios y en la desinformación ciudadana. La profusión de autodenominados gurús o sanadores que apelan a la autosugestión contribuye a la confusión y al culto no saludable de lo saludable.

Palabras claves. Salud, holismo, mercantilismo, abuso institucionalizado, educación, ortorexia, ética del cuidado

Abstract. This paper aims at claiming the ancestral wisdom of indigenous people in the health area. It analyzes how health has been commodified in the interest of large companies (particularly those related to the pharmaceutical industry) to the detriment of a holistic definition of wellness through education. Furthermore, the concept of health as a right disagrees with such commodification or sale to the highest bidder, which prompts dehumanization of health services and public misinformation. The abundance of self-proclaimed gurus or healers who appeal to autosuggestion contributes both to confusion and to an unhealthy cult of healthiness.

Keywords. Health, holism, commodification, institutionalized abuse, education, orthorexia, ethics of care.

\footnotetext{
${ }^{1}$ Licenciado en Economía por la Universidad de Barcelona (España) y University of Southampton (Reino Unido). Doctorado en Educación con Especialidad en Mediación Pedagógica por la Universidad La Salle, Costa Rica.
} 
doi: http://dx.doi.org/10.15359/ree.19-3.20

URL: http://www.una.ac.cr/educare

CORREO: educare@una.cr

Cuando Sibö creó el mar, también creó las semillas. Hizo varias pruebas con granos de maíz, pero las semillas no podían germinar sin ayuda del murciélago. De una semilla nacieron los indígenas allá donde sale el sol. Otra semilla la puso en una balsa y le sopló, la balsa se perdió en el mar. Era la semilla de los sikuas, de los extranjeros con piel y rasgos distintos que después volverían, como las tortugas cuando desovan, traídos por el mar.

Relato de la tradición oral indígena bribri (Sánchez, 1997)

\section{El rapto de Sibö}

Sibö (en bribri, Sib `ö), dios padre y creador de la tierra y de los humanos - a quienes enseñó qué productos comer, cómo sembrar y qué plantas cultivar- según los bribris, habitantes de las tierras de Talamanca (actualmente territorio costarricense), ha sido secuestrado. Surá, contraparte inseparable de Sibö, útero de toda vida, guardiana de las semillas que simbolizan el alma -por ser la tierra misma y quien da forma a la humanidad como excelsa artesana-corre grave peligro.

Lo más estremecedor es que el rapto de marras ha sido perpetrado por los mismos hijos de la divinidad que los creó. La práctica de la biopiratería en Costa Rica (Ortega, 2009), arteramente auspiciada por las mismas autoridades del país, supone un caso paradigmático en el mundo de hasta qué punto los intereses mercantiles y el escandaloso beneficio económico de unos pocos en detrimento del bien de muchos puede arrasar la sabiduría ancestral de todo un pueblo -en este caso, los indígenas costarricenses- y vender el valor sagrado de la salud al mejor postor dispuesto a profanarlo.

Costa Rica-en cuyo territorio (menos del $0,1 \%$ de la masa terrestre del planeta) se concentra el $6 \%$ de la biodiversidad mundial (Hickman, 2007)- aprobó la Ley de Biodiversidad en 1998, un eficaz marco legal de protección frente a los bioprospectores (eufemismo de biopiratas), representantes de industrias farmacéuticas cuya misión consiste en extraer información a los nativos sobre el uso de plantas con vistas a aislar químicamente sus moléculas activas en concentrados y así comercializarlos, obteniendo ingresos millonarios sin revertir nada en sus indefensos benefactores; esto es, se normó el encuentro entre la cultura de la generosidad (indígena) y la de la rapiña (farmacéutica).

Sin embargo, el Tratado de Libre Comercio firmado con Estados Unidos en 2004 y ratificado con un estrecho margen por referéndum popular en 2007 modificó, e incluso invalidó, muchas de las disposiciones de la Ley de la Biodiversidad, de tal modo que la población indígena volvía a quedar inerme frente a una maraña legal de patentes que la utilizaba para la generación de remedios médicos y, al mismo tiempo, la excluía de cualquier tipo de compensación.

Por otra parte, los referentes históricos de un país juegan un rol determinante a la hora del diseño y ejecución de sus políticas. El Gobierno actual de Costa Rica dio la espalda a su población indígena ignorando la recomendación de la Sala Constitucional y violando el convenio № 169 de la 
doi: http://dx.doi.org/10.15359/ree.19-3.20

URL: http://www.una.ac.cr/educare

CORREO: educare@una.cr

Organización Internacional del Trabajo (OIT) (1989) al prescindir de la obligada consulta indígena vía decreto presidencial ${ }^{2}$, un remedo de las bases constitutivas y los signos identitarios del país:

De muchas maneras la Sociedad, el Estado y la República de Costa Rica fueron inventados, diseñados y construidos sin necesidad de contar con el apoyo o el rechazo de los indios que habitaban en el Valle Central o que vivían en la Cordillera de Talamanca. La nacionalidad costarricense negó entonces al indio y trató de borrar todo vestigio o seña que pudiera evidenciar un pasado indígena. (Borge, 1998, p. 346)

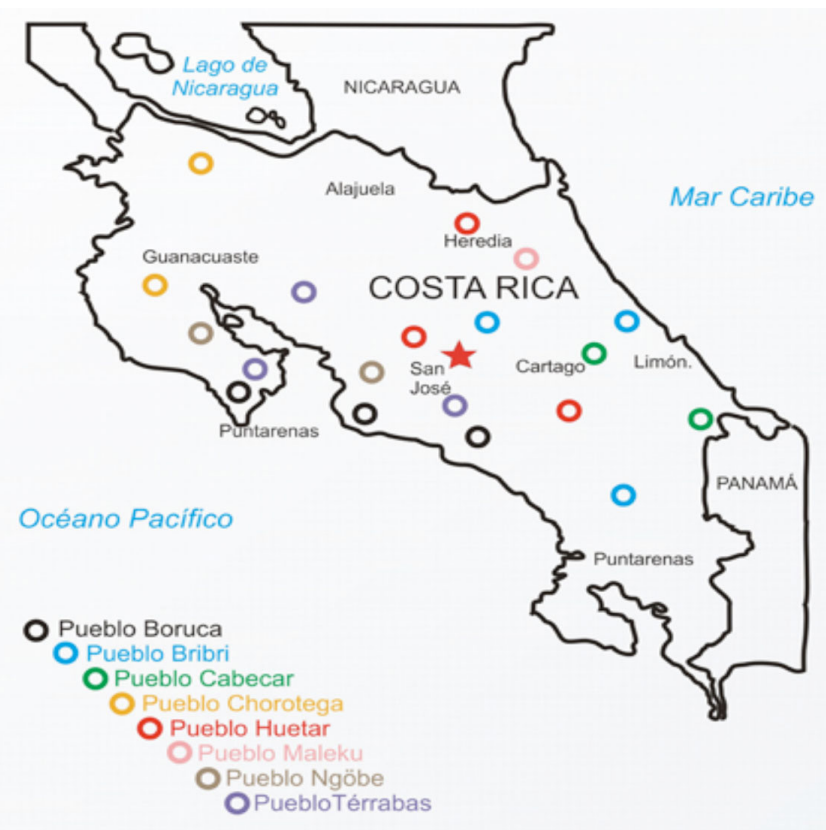

Figura 1. Pueblos indígenas de Costa Rica. UNESCO (s. f.).

La población indígena de Costa Rica, integrada por ocho pueblos distribuidos en veinticuatro territorios (véase figura 1), con aproximadamente setenta mil personas, no cuenta con representación política en el Congreso; su esperanza estriba en la auto-organización de sus comunidades -su reinvención autopoiética (Maturana y Varela, 2004)-, la creación de sinergias a través de redes de informacióny apoyo yel recurso de mediación de entidades internacionales quevelen efectivamente por sus derechos.

\footnotetext{
${ }^{2}$ Ley no 7788 -Ley de Biodiversidad de 23 de abril de 1998 enmendada por Ley 8686 - Reforma, Adición y Derogación de varias normas que regulan materias relacionadas con Propiedad Intelectual de 26 de noviembre de 2008 (OMPI, 2008).
} 
doi: http://dx.doi.org/10.15359/ree.19-3.20

URL: http://www.una.ac.cr/educare

CORREO: educare@una.cr

La salud, por lo tanto -al igual que otras áreas fundamentales en el desarrollo humano como la educación y la cultura-, ha sido alcanzada por los tentáculos del mercado y tratada como negocio según la siguiente tríada de objetivos vertebradores:

1) Reducción de costos.

2) Ampliación del público objetivo.

3) Incremento de los márgenes de ganancia.

Maximización del beneficio

\section{Crónica de una crisis anunciada}

En este sentido, la conocida crisis de la Caja Costarricense de Seguro Social (CCSS) (Badilla y Díaz, 2013) no se relaciona con la incapacidad del Estado para proveer servicios de salud de calidad a la ciudadanía por falta de recursos, sino con la mala gestión y la corrupción consentida -y diríase que deliberada- de ese mismo Estado que durante casi cuarenta años incumplió sistemáticamente sus obligaciones de pago, activando -ya desde entonces- la bomba de relojería que hoy detona con tanta y tan anticipada virulencia; no se trata, por lo tanto, de un problema por exceso de gastos necesarios (excusa muy conveniente para recortarlos, dar alas a la medicina privada y marginar a la población sin recursos), sino por defecto de ingresos debitados (una verdad incómoda donde las haya que pone sobre el tapete el desprecio hacia cualquier consideración ética de los gobernantes a quienes se les encomendó salvaguardar la salud pública).

Rodrigo Arias López, matemático actuarial que trabajó en la Dirección Actuarial de la CCSS, advirtió en vano desde el 2004 a la Junta Directiva de dicha entidad sobre el déficit que hoy sufre:

Ahora la OPS (Organización Panamericana de la Salud) nos dice que la crisis es culpa de la Sala [Constitucional], por considerar la salud como un derecho [negrita añadida]. El problema no es ese. La posición de la OPS lo que hace es salvar a los corruptos al enfocarse en los gastos, pero respecto a los ingresos dice que "no se puede hacer nada". (Córdoba, 2011)

En realidad, esta crisis es un reflejo sintomático de otra mucho más profunda, una crisis raíz de valores que afecta al conjunto de la sociedad -especialmente en un contexto de globalización donde el efecto mariposa de Lorenz (Briggs y Peat, 1999) es cada vez más evidente en todos los campos-y que se materializa en una dolorosa fragmentación a todos los niveles. Tal como sostiene Morin (2002, p. 2): 
Las especializaciones en todos los sectores económicos del trabajo y del pensamiento también, encierran a los seres humanos en actividades fragmentadas, aisladas y donde se pierde el sentido de la realidad común en la cual nosotros estamos incluidos. De este modo el sentido de la responsabilidad [negrita añadida] para los otros y para su comunidad, también se desintegra. No olvidemos, además que los motores del desarrollo en la ciencia, la técnica, la economía, el provecho están sin ningún control ético.

La salud, concebida holísticamente como bienestar integral -fusionando los ámbitos físico y mental seccionados desde la segunda mitad del S.XVII hasta finales del S.XIX por la típicamente cartesiana concepción dualista del ser-, se ha erigido en poco menos que tótem digno de culto público y privado: no solo ocupa lugar preferente en la agenda política tanto como garantía social y recurso de movilización en la intención de voto (si bien la amenaza de la privatización se cierne sobre aquella y, por lo tanto, restringe las opciones reales de esta), sino que se ha colado en la intimidad, arrogándose la facultad de traducir ex cátedra, desde el exterior, el estado interior de cualquier persona.

El aluvión de bibliografía y el incesante cubrimiento mediático al respecto confirman esta tendencia al alza; no obstante, mal que le pese al aura casi mística que adorna las formas del debate, la superficialidad de fondo aparece como una constante en el abordaje de la cuestión. ¿Es posible desentrañar la complejidad de la salud, inmersos en el frenesí de la sobreinformación, sin caer en la trivialidad? Y, sobre todo, ¿hasta qué punto estamos aprendiendo sobre el tema o simplemente estamos siendo aleccionados?

Educación y salud van de la mano por cuanto la primera mediatiza la percepción (Capra, 1998) -convenientemente maleable- que tenemos de la segunda, cada vez más homogénea en virtud de un consenso universal acerca de lo bueno y lo malo atávicamente polarizador, una división maniquea que no deja de resultar chocante en un escenario donde paradójicamente se ensalzan las bondades de la unidad entre materia y espíritu como paradigma de pretendida modernidad ${ }^{3}$.

La ubicuidad de los medios de comunicación de masas -y, concretamente, la televisión ${ }^{4}-$ ha propiciado que estos hayan asumido, por derecho propio, un rol educador preponderante en la sociedad, de tal modo que la dimensión visual y la interacción digital se han aliado para ofrecer -a través de una convergencia simplificadora- respuestas "exprés" a toda clase de preguntas; así las cosas, se difuminan los límites de la disciplina en beneficio de herramientas promocionales al servicio de una uniformidad cultural sospechosamente parecida al pensamiento único de los regímenes totalitarios.

\footnotetext{
${ }^{3}$ La milenaria cita latina de Juvenal, mens sana in corpore sano, nos recuerda la larga -y tan a menudo olvidadahistoria de tan conveniente maridaje.

${ }^{4}$ La media diaria de horas ante la televisión por persona en Estados Unidos es de 5 horas y 11 minutos (The Nielsen Company, 2010).
} 
doi: http://dx.doi.org/10.15359/ree.19-3.20

URL: http://www.una.ac.cr/educare

CORREO: educare@una.cr

Chaplin (2010-2011, pp. 2-3) pone sobre el tapete la inevitable frivolidad de la televisión como instrumento de divulgación científica:

Las constricciones temporales y estructurales que rigen la televisión se oponen a la comunicación del pensamiento complejo. ... Apresurada y superficial por definición, según sigue el argumento, la televisión fomenta la urgencia, ansía "comida cultural rápida" y a la fuerza ahoga toda discusión seria. La conclusión es que la televisión obliga a los filósofos a reinventarse como meros propagandistas, que resumen su obra en extractos formularios y en lemas vulgarizadores, o bien como groseros publicistas que hablan de sus libros para aumentar las ventas.

El sociólogo Bourdieu (1998, p. 39) expresa esta misma inquietud sucinta y lúcidamente: “¿Se puede pensar atenazado por la velocidad? ¿Acaso la televisión, al conceder la palabra a pensadores supuestamente capaces de pensar a toda velocidad, no se está condenando a no contar más que con fast thinkers, con pensadores que piensan más rápido que su sombra...?"

Ensartado entre los alfileres paralizantes de semejante hermenéutica, el mismo concepto de salud es susceptible de chalaneo como valor comercial. La formación específica y la red de intereses de quienes contribuyen a formar opinión pasan a un segundo plano -en aras de la transmisión de un mensaje sesgado e inculcado a machamartillo- para asegurar su raigambre en el inconsciente colectivo, de tal modo que contravenirlo se considera herejía. Las nuevas plataformas de difusión de dicho mensaje sofistican viejos métodos de persuasión (léase entorno de totalitarismo cibernético [Lanier, 2007] o maoísmo digital) y la figura del gurú -cajón de sastre de charlatanes y arribistas de la peor ralea, por más credenciales que los avalen- deviene poco menos que sacrosanta.

\section{Salud y culpa}

Como botón de muestra, los médicos Larry Dossey y Deepak Chopra representan dos vertientes opuestas de este fenómeno, constatables a partir del análisis que cada uno elabora de un mismo experimento para medir la repercusión de las enfermedades cardíacas citado en sus respectivas obras (Chopra, 1992; Dossey, 2004): en la Universidad de Ohio, durante la década de los setenta del siglo XX, varios grupos de conejos fueron sometidos a una dieta rica en colesterol con los devastadores efectos esperados en el sistema arterial, excepto en aquellos que, antes de ser alimentados, fueron acariciados y sostenidos amorosamente por uno de los cuidadores; es decir, el trato humanitario incidía directamente en la resistencia a la ingesta tóxica. 
Mientras Chopra5(1992) achaca tal anomalía a una inmunidad operada por el cerebro del conejo ante la estimulación recibida -concluyendo que uno mismo puede auto-generarse poder mental a discreción (eso sí, siguiendo al pie de la letra una serie de farragosas recomendaciones "curativas" que pasan, casualmente, por consumir los productos que este autor comercializa o ingresar en la clínica que regenta)-, Dossey (2004) llega a una inferencia mucho más verosímil y políticamente incorrecta: después de todo, tal vez la alimentación y otras pautas dictadas por la nueva religión de lo saludable no revistan la importancia que se les adjudica en contacto con realidades mucho más profundas $-\mathrm{y}$, por ende, menos comercializables- como las diferencias genéticas, las experiencias tempranas en un plano que no podemos controlar, el tesón en priorizar aquello que nos apasiona y la gran capacidad de adaptación con la que nuestro organismo ha sido dotado en el plano de aquello en lo que sí nos es dado terciar.

Si planteáramos este caso a la inversa, no sería descabellado concluir que una dieta perfectamente equilibrada en cuanto a calidad orgánica, lapso entre comidas y componentes vitamínicos personalizados -según los requerimientos calóricos por edad, sexo y nivel de sedentarismo- podría no reportar las tan favorables repercusiones que el dogma naturista le atribuye en cuanto al bienestar integral del individuo. Criticando el sistema cartesianonewtoniano, ¿no estaremos incurriendo en el mismo error al disgregar el qué del cómo en la alimentación? No basta con cumplir ciertos requisitos externos cuyo seguimiento extremo conduce a la esclavitud (véase la patología de la ortorexia ${ }^{6}$ [Bratman y Knight, 2001]): es esencial cultivar la motivación interna como camino a la libertad y a la autorrealización ${ }^{7}$ (Gandhi, 2004). Thomas Edison (Dossey, 2004) y Julio Verne (Mayor, 2004), por ejemplo, compartían terribles hábitos alimenticios, pero la pasión que movía a ambos constituía -por encima de cualquier suplemento nutricional- la clave de su potente motor vital.

El hambre epidérmica es bien conocida en el mundo infantil: la falta de contacto corporal origina depresión y, en casos extremos, la muerte. Los estudios realizados al respecto por Spitz (1945) y Bowlby (1951) en orfanatos donde bebés bien alimentados y limpios experimentaban estos procesos por falta de contacto corporal afectivo avalan la equivalencia entre tacto y vida en la infancia temprana. ¿Hemos olvidado por el camino hacia el progreso este vínculo sagrado como epítome de salud, o tal vez hemos sido forzados a la amnesia por un sistema que se fortalece con la desmemoria colectiva?

${ }_{5}$ Deepak Chopra cobra $\$ 25.000$ dólares por conferencia, donde brinda consejos espirituales y advierte paradójicamente sobre los efectos negativos del materialismo (para ver su montaje comercial, consultar la página web: www.chopra.com).

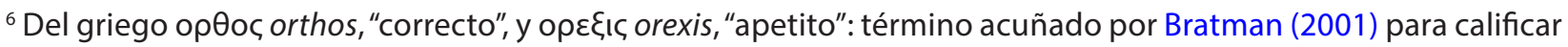
como trastorno alimentario la obsesión de ingerir comida considerada saludable como una obsesión perjudicial para la salud.

${ }^{7}$ En el sentido explicado por Gandhi: la acción desinteresada. 
doi: http://dx.doi.org/10.15359/ree.19-3.20

URL: http://www.una.ac.cr/educare

CORREO: educare@una.cr

Lo más curioso del experimento evocado por Dossey (2004) y Chopra (1992), sin embargo, es lo que ninguno de los dos menciona y, lo que es más grave, lo que ni siquiera parece sorprender a estos adalides de la medicina holística: ¿por qué solo una de las personas encargadas de alimentar a los conejos tuvo un trato humanitario con ellos?, ¿acaso la frecuente despersonalización al interrelacionarnos con los demás seres vivos nos ha insensibilizado hasta el punto de no detectar lo anormal de cuanto normalizamos cotidianamente?

En este sentido, la ética del cuidado suscrita por Lobo $(2002$, p.6) adquiere pleno significado:

La ética del cuidado la disfrutamos en nuestros primeros años de vida y luego la abandonamos por la ética de la justicia. La justicia es bipolar, las cosas son buenas o malas, justas o injustas, correctas o incorrectas. Premio y castigo. La ética de la justicia es la columna vertebral del sistema patriarcal.

El Dr. Dossey pone el dedo en la llaga de la deshumanización que asuela el campo sanitario al denunciar el "abuso institucionalizado" que soporta el estudiantado de medicina y que, en el colmo del cinismo, reproduce desde una perspectiva casi cómica -haciendo tolerable lo intolerable al desdramatizar el ejercicio sistemático de la humillación- multitud de series de televisión ${ }^{8}$. Es sobradamente conocido el efecto imitación que se deriva de tales programas al concitar voluntades de jóvenes egocéntricos cuya máxima motivación es emular las vidas ficcionales de sus protagonistas, relegando la vocación real a mera anécdota intrascendente. En un estudio efectuado por la Universidad de Zaragoza, el 11\% de estudiantes de medicina reconocía haberse decidido por esta especialidad influenciado por las series de televisión sobre médicos (Soria, Guerra, Giménez y Escanero, 2006).

\section{Educación para la salud integral}

La mitología griega (Graves, 2007) reconoce a Higía como diosa de la curación, representada a menudo con un cáliz (símbolo del remedio) del que bebía una serpiente (símbolo de rejuvenecimiento por su periódica muda de piel); así las cosas, la llamada copa de Higía se ha convertido en el distintivo más difundido internacionalmente de la profesión farmacéutica.

Considerando el sombrío panorama señalado en los puntos anteriores sobre la mercantilización de la medicina, agravado por su uso fraudulento como herramienta de control social y, por lo tanto, como peligrosa arma política de dominación, cabe replantearse el significado de estos signos transmutados en amenaza a la salud e incluso a sus fundamentos epistemológicos por obra y gracia de la plusvalía: el veneno que salva aplicado en pequeñas dosis al generar anticuerpos en el organismo que lo recibe es el mismo que, sin la medida adecuada, lo destruye.

${ }^{8}$ Por citar algunas de las más conocidas: Anatomía de Grey, Dr. House, Hospital Central, MIR y Urgencias.

8 
El excelente ensayo de Blench (2005), Los inventores de enfermedades, denuncia el negocio de la medicina y hace temblar, entre otros, los frágiles cimientos de la psiquiatría. Si al terminar la Segunda Guerra Mundial existían 26 enfermedades mentales consignadas, hoy se registran casi 400, un hiperbólico aumento de prácticamente un $1.500 \%$ en apenas 66 años. ¿La humanidad en conjunto se ha puesto de acuerdo para volverse loca, o estamos al albur de quienes diseñan y encasquetan a la población pseudoenfermedades (avaladas por la poderosa industria farmacéutica que, muy oportunamente, posee un remedio específico para cada nuevo mal declarado)?

La educación refleja y reproduce estas construcciones sociales y las inculca, implícita o explícitamente, a través de contenidos que devienen pretextos de idearios muy a menudo perjudiciales para la salud integral de quienes los reciben. La trampa del pensamiento positivo, sin ir más lejos, es causa de muchos sufrimientos:

Las víctimas de las crisis no solo tienen que sufrir en silencio su desgracia, sino que casi se ven obligadas a estar contentas. ... Uno de los argumentos falaces que emplean los positivistas es dividir el mundo entre los que piensan en positivo como ellos y los pesimistas depresivos. (Muñoz, 2011, párr. 2 y 12)

El "John Henrismo" que menciona Dossey (2004) como responsable de expectativas desmesuradas en relación con el esfuerzo invertido para hacerlas realidad tiene mucho que ver con la desmotivación y la frustración que atormenta a tantas personas jóvenes -y no tan jóvenes- en etapa escolar, colegial o universitaria.

El rígido ambiente de tantos centros educativos en cuanto a horarios y actividades obligatorias deja poco margen a la creatividad y a las energías necesarias para desarrollarla, de ahí que la quinta dimensión a la que se refiere Payán (2000) -la conciencia- adquiera importancia capital como recordatorio de nuestra realidad profunda más allá de las limitaciones tangibles. En este sentido, las aportaciones individuales hacia la innovación y la mejora, por modestas que resulten, siempre acaban sumando y tienen un efecto multiplicador (el experimento del "centésimo mono" de Ken Keyes Jr. lo ejemplifica claramente):

Aunque el número exacto puede variar, el fenómeno del centésimo mono significa que cuando un número limitado de individuos conoce un nuevo método, solo es propiedad consciente de tales individuos; pero existe un punto (masa crítica) en el que con una persona más que se sintonice con el nuevo conocimiento, éste llega a todo el mundo (eclosión ideológica).... Podríamos decir que el mono número cien permitió que aflorara la quinta dimensión. (Payán, 2000, p. 35)

La educación integradora abarca, valga la redundancia, la salud integral del individuo y del entorno que lo acoge, desde sus posibilidades y como semilla hacia ese "tomar conciencia" 
doi: http://dx.doi.org/10.15359/ree.19-3.20

URL: http://www.una.ac.cr/educare

CORREO: educare@una.cr

(Coles, 1998, p. 115) como misión pedagógica fundamental enfocada hacia la autonomía y la solidaridad (bases ontológicas y físicas de la salud). Las instituciones o sistemas donde se lleva a cabo la educación (academia) o curación (centro de salud) de las personas guardan estrechos paralelismos con las estructuras disipativas estudiadas por Prigogine (1983) y las implicaciones que de las mismas señala Dossey (1986) en la salud de los seres humanos: si en el ámbito de la física queda demostrado que del orden surge el caos -aun más, sin caos no podría surgir el orden-, del mismo modo en un ámbito de relaciones humanas presididas por el bien común, más allá de la burocracia que lo pone en entredicho, pueden surgir órdenes extraordinarios: la canalización de manera visible de talentos ya existentes.

De acuerdo con Dossey (1986), la clave del crecimiento es la fragilidad, así que unas condiciones adversas como punto de partida no solo no deberían conducir al desánimo, sino considerarse incluso como caldo de cultivo idóneo para el surgimiento de un nuevo y mejor orden que -como recuerda Maturana (1999)- implique a toda la comunidad.

Por consiguiente, quien quiere acierto sin error, orden sin desorden, no comprende los principios

por los que se rigen el cielo y la tierra.

No entiende cómo

todo está conectado.

Chuang Tzu (S. IV a. C.)

\section{Referencias}

Badilla, A. y Díaz, P. (Julio-Diciembre, 2013). Caja Costarricense de Seguro Social: Variables internas que perpetúan la crisis. Revista Rupturas, 3(2), 202-223. Recuperado de http://investiga. uned.ac.cr/rupturas/

Blench, J. (2005). Los inventores de enfermedades. Cómo nos convierten en pacientes. Barcelona: Destino.

Borge, C. (1998). Los indios de Costa Rica: De su negación a su reivindicación. En M. E. Bozzoli, R. Barrantes, D. Obando y M. Ropjas (Comps.), Primer congreso científico sobre pueblos indígenas de Costa Rica y sus fronteras. Memoria (pp. 346-359). San José, Costa Rica: EUNED, Unicef y Universidad de Costa Rica.

Bourdieu, P. (1998). Sobre la televisión (2ª ed.). Barcelona: Anagrama.

Bowlby, J. (1951). Cuidado maternal y salud mental. Ginebra: World Health Organization. 
Bratman, S. y Knight, D. (2001). Adictos de la comida sana. Ortorexia nerviosa: superando la obsesión con la alimentación saludable. Nueva York: Crown Publishing House.

Briggs, J. y Peat, D. F. (1999). Las 7 leyes del caos. Barcelona: Grijalbo.

Capra, F. (1998). La trama de la vida. Una nueva perspectiva de los sistemas vivos. Barcelona: Anagrama.

Chaplin, T. (2010-2011). La filosofía en televisión: ¿Un sueño imposible?. Barcelona: Museu d'Art Contemporani de Barcelona (MACBA) y Centro Galego de Arte Contemporánea (CGAC). Recuperado de http://www.macba.cat/uploads/TWM/TV chaplin cas.pdf

Chopra, D. (1992). La curación cuántica. Explorando las fronteras de la medicina mental y corporal. Barcelona: Grijalbo.

Coles, R. (1998). La inteligencia moral del niño y del adolescente. Barcelona: Kairós.

Córdoba, J. (20 de julio de 2011). Caja tendría superávit de $\mathbb{\$} 30.000$ millones si el Estado pagara anualmente sus cuotas. Semanario Universidad. Recuperado de http:// semanariouniversidad.ucr.cr/pais/caja-tendra-supervit-de-30000-millones-si-el-estadopagara-anualmente-sus-cuotas/

Dossey, L. (1986). Tiempo, espacio y medicina. Barcelona: Kairós.

Dossey, L. (2004). El poder curativo de la mente. La salud más allá del cuerpo. México: Alamah.

Gandhi, M. (2004). El Bhagavad Guita de acuerdo a Gandhi. Buenos Aires: Kier.

Graves, R. (2007). Los mitos griegos. Barcelona: Ariel.

Hickman, L. (26 de mayo de 2007). Shades of green [Tonos de verde.] The Guardian. Recuperado de http://www.theguardian.com/travel/2007/may/26/saturday.costarica

Lanier, J. (2007). La mitad de un manifiesto. En J. Brockman (Ed.), El nuevo humanismo y las fronteras de la ciencia (pp. 291-325). Barcelona: Kairós.

Lobo, T. (Noviembre, 2002). Abordar la historia desde la ficción literaria (o cómo destejer la bufanda). Revista Comunicación, 12(23), 1-8. Recuperado de http://revistas.tec.ac.cr/index. php/comunicacion/article/view/1241/1145

Maturana, H. (1999). Transformación en la convivencia. Santiago, Chile: Dolmen Ediciones.

Maturana, H. y Varela, F. (2004). De máquinas y seres vivos. Autopoiesis: La organización de lo vivo ( $\left.6^{\mathrm{a}} \mathrm{ed}.\right)$. Buenos Aires: Lumen. 
doi: http://dx.doi.org/10.15359/ree.19-3.20

URL: http://www.una.ac.cr/educare

CORREO: educare@una.cr

Mayor, D. (2004). Julio Verne: Grandes biografías. Madrid: Edimat.

Merton, T. (1969). The Way of Chuang Tzu [El camino de Chuang Tzu]. Nueva York: New Directions.

Morin, E. (5 y 6 de septiembre de 2002). Ética y globalización. Seminario internacional: Los desafíos éticos del desarrollo. Buenos Aires: Universidad de Buenos Aires. Recuperado de http:// www.ucipfg.com/Repositorio/MCSH/MCSH-01/1.BARCELONA/BLOQUE-ACADEMICO/ Unidad-2/lecturas-Complementarias/news21 morin.pdf

Muñoz, R. (17 de julio de 2011). Optimistas a la fuerza, pase lo que pase. El País. Recuperado de http://elpais.com/diario/2011/07/17/sociedad/1310853601 850215.html

Organización Internacional del Trabajo (OIT). (1989). Convenio № 169. Recuperado de http:// www.ilo.org/indigenous/Conventions/no169/lang--es/index.htm

Organización Mundial de la Propiedad Intelectual (OMPI). (26 de noviembre de 2008). Ley № 7788 de Biodiversidad de 23 de abril de 1998 enmendada por Ley 8686 Reforma, Adición y Derogación de varias normas que regulan materias relacionadas con Propiedad Intelectual. Recuperado de http://www.wipo.int/wipolex/es/details. jsp?id=9019

Ortega, P. (2009). El rapto de Sibö. Crónicas de biopiratería en Costa Rica [CD]. San José, Costa Rica: Unidad de Producción Audiovisual de la Vicerrectoría de Acción Social de la Universidad de Costa Rica.

Payán, J. C. (2000). Lánzate al vacío, se extenderán tus alas. Bogotá: McGraw-Hill.

Prigogine, I. (1983). ¿Tan solo una ilusión? Barcelona: Tusquets.

Sánchez, J. (1997). 'Relatos bribris de Keköldi, Provincia de Limón, Costa Rica'. Tradición oral indígena costarricense, 4(1-2), 1-83.

Soria, M., Guerra, M., Giménez, I. y Escanero, J. F. (Junio, 2006). La decisión de estudiar medicina: Características. Revista Educación Médica, 9(2), 91-97. Recupera de http://scielo.isciii.es/ pdf/edu/v9n2/original4.pdf

Spitz, R. A. (1945). Hospitalism [Hospitalización]. En R. A. King, S. Abrams, A. S. Dowling y P. M. Brench. The Psychoanalytic Study of the Child [Estudio psiquiátrico del niño] (Vol. 1). Nueva York: International Universities Press. 
The Nielsen Company. (2010). State of the Media Trends in TV Viewing-2011 TV upfronts [Estado de tendencias de los medios de comunicación en audiencias de TV-avances de TV 2011]. Recuperado de http://www.nielsen.com/content/dam/corporate/us/en/newswire/ uploads/2011/04/State-of-the-Media-2011-TV-Upfronts.pdf

UNESCO. (s. f.). Descripción resumida del pueblo cabécar. Recuperado de http://www.unesco.org. uy/phi/aguaycultura/es/paises/costa-rica/pueblo-cabecar.html

\section{Cómo citar este artículo en APA:}

de Muller, A. (Setiembre-Diciembre, 2015). Transformación personal y social en el ámbito de la salud a través de la educación: Breve recorrido desde la sabiduría indígena ancestral hasta la tiranía moderna de lo saludable. Revista Electrónica Educare, 19(3), 1-13. doi: http://dx.doi.org/10.15359/ree.19-3.20

Nota: Para citar este artículo en otros sistemas puede consultar el hipervínculo "Como citar el artículo" en la barra derecha de nuestro sitio web: http://www.revistas.una.ac.cr/index.php/EDUCARE/index 